\title{
Induced neuroprotection by remote ischemic perconditioning as a new paradigm in ischemic stroke at the acute phase, a systematic review
}

Francisco Purroy ${ }^{1,2^{*+}}$ D C Cristina García ${ }^{2}$, Gerard Mauri ${ }^{1,2}$, Cristina Pereira ${ }^{2}$, Coral Torres², Daniel Vazquez-Justes ${ }^{1,2}$, Mikel Vicente-Pascual ${ }^{1,2}$, Ana Vena ${ }^{1,2}$ and Gloria Arque ${ }^{2^{*+}}$

\begin{abstract}
Background: Remote ischemic conditioning during cerebral ischemia (remote ischemic perconditioning, RIPerC) refers to the application of several cycles of brief ischemia and reperfusion (I/R) commonly to a limb, and it represents a new paradigm in neuroprotection with multiple mechanisms of action in ischemic stroke (IS) patients during acute phase. Some clinical trials just finished, and a few others are still ongoing; gather the current knowledge and pull it down to influence the present and future studies was the goal of this paper.

Methods: A systematic review of published research papers and/or registered clinical trials since 2000 was performed.

Results: Nineteen studies were identified and only four studies were completed. All of them have demonstrated that RIPerC is safe, feasible and well tolerated in IS patients. However, a high heterogeneity of clinical trial characteristics was observed: five (26.3\%) randomized clinical trials (RCTs) included only thrombolytic-treated patients, three (15.8\%) RCTs only thrombectomy-treated patients, and five (26.3\%) RCTs required radiological confirmation of IS. Temporal inclusion criteria vary from $4 \mathrm{~h}$ to $48 \mathrm{~h}$. Most of the clinical trials used 4 cycles of RIPerC in the upper non-affected limb. Interestingly, only three (16.7\%) RCTs applied RIPerC during the transportation in the ambulance. Neuroimaging outputs were the main endpoints when endovascular therapy was applied; functional outcome is also the main endpoint in large-medium size studies.

Conclusions: This review summarizes the completed and ongoing clinical trials on RIPerC in IS patients, where RIPerC has been used alone or in combination with recanalization therapies. Ongoing clinical trials will provide new information on the best RIPerC intervention strategy and potentially improve the functional outcome of IS patients; definition of new RIPerC strategies would ideally aim at enhancing tissue preservation, promoting neurological recovery, and stratify patients to improve treatment feasibility.
\end{abstract}

Keywords: Ischemic stroke, Neuroprotection, Remote ischemic perconditioning, Randomized clinical trials, Systematic review

\footnotetext{
*Correspondence: fpurroygarcia@gmail.com; gloria.arque@gmail.com

${ }^{\dagger}$ Francisco Purroy and Gloria Arque contributed equally to this work.

'Stroke Unit, Department of Neurology, Universitat de Lleida, Hospital

Universitari Arnau de Vilanova, Avda Rovira Roure 80, 25198 Lleida, Spain

${ }^{2}$ Clinical Neurosciences Group, Institut de Recerca Biomèdica de Lleida

(IRBLleida). UdL, Lleida, Spain
}

(c) The Author(s). 2020 Open Access This article is licensed under a Creative Commons Attribution 4.0 International License, which permits use, sharing, adaptation, distribution and reproduction in any medium or format, as long as you give appropriate credit to the original author(s) and the source, provide a link to the Creative Commons licence, and indicate if changes were made. The images or other third party material in this article are included in the article's Creative Commons licence, unless indicated otherwise in a credit line to the material. If material is not included in the article's Creative Commons licence and your intended use is not permitted by statutory regulation or exceeds the permitted use, you will need to obtain permission directly from the copyright holder. To view a copy of this licence, visit http://creativecommons.org/licenses/by/4.0/ The Creative Commons Public Domain Dedication waiver (http://creativecommons.org/publicdomain/zero/1.0/) applies to the data made available in this article, unless otherwise stated in a credit line to the data. 


\section{Background}

Stroke is one of the leading causes of death and disability worldwide [1], with 10.3 million of new strokes and 113 million of disability-adjusted life years per year [2]. Stroke victims face an uncertain future and a life severely affected by disability. The most common type of stroke is the ischemic stroke (IS), accounting for $87 \%$ of all strokes. It is characterized by the occlusion within an arterial vessel supplying blood to an area of the brain, resulting in a corresponding loss of neurological function. It mainly occurs in elderly patients of both sexes with often multiple comorbidities (diabetes mellitus, hypertension, hyperlipidemia, obesity) [3]. Currently, the only treatments available in the acute phase that have demonstrated safety and effectiveness are intravenous fibrinolytic treatment $[4,5]$ and mechanical thrombectomy [6]. Unfortunately, even today many patients cannot benefit from these treatments due to contraindications, time of evolution of the symptoms or restricted access to mechanical therapies that are currently only offered in highly sophisticated hospitals. Thus, there is a need for better and wider therapies to boost patient adherence.

The effectiveness of neuroprotective therapies has a great potential to not only increase the benefits of available reperfusion therapies but also to provide an advisable medical procedure for patients who are not eligible for current treatments. However, translation of most neuroprotective trials from the bench to the emergency room has failed so far, they did not demonstrate efficacy on IS patients, even with promising results in a few preclinical studies [7]. One of the main explanations for this failure is that the majority of neuroprotective drugs studied only act on a level of the complex cascade of phenomena that occur in ischemia/reperfusion [7-9]. The feasibility of neuroprotection in IS is still an unresolved inquiry. To date, all trials of neuroprotectant compounds have failed to provide basis and build better trials.

Remote ischemic perconditioning (RIPerC) represents a new paradigm in neuroprotective therapies $[10,11]$ and it has the potential ability to protect the ischemic brain from injury until reperfusion and, later to protect the brain from reperfusion injury. RIPerC consists of short and controlled cycles of ischemia-reperfusion applied to one limb during the establishment of cerebral ischemia [11]. Until now, the underlying mechanisms of remote ischemic conditioning (RIC) include neurovascular protection, induced anti-inflammatory action and neuronal protection against excitotoxicity; paired together with mitochondrial protection, circulating inflammasome activation and/or transcriptional regulation of neuroprotective pathway [12] (Fig. 1). However, there is limited data about the clinical translation of RIPerC in IS patients.
Endogenous cerebral neuroprotection of RIPerC on IS patients is a new paradigm that aims to enhance the brain resilience to ischemia and it has the potential to improve the clinical outcome of affected individuals. For that, a systematic review of the published articles and clinical trials on RIPerC applied to IS patients was performed to evaluate and summarize the findings.

\section{Search strategy and selection criteria}

A systematic review of prospective cohort studies was conducted (prehospital-based and hospital-based cohorts) on acute IS patients under RIPerC and placeboarms. Studies published from January 2000 to March 2020 were included. PRISMA recommendations were followed [13].

Identification, screening and eligibility for included studies was performed by two reviewers (F.P., G.A.). Bias analysis was unable to be performed because of the ongoing clinical trials. The search was conducted using the electronic databases: Pubmed and ClinicalTrials.gov. Search limits were English language, human and 2000current. The search terms were: 'remote ischemic conditioning' AND 'acute ischemic stroke' OR 'remote ischemic perconditioning' AND 'acute ischemic stroke' OR 'remote ischemic postconditioning' AND 'acute ischemic stroke'. Prospective human cohort studies that applied RIPerC in IS patients were included. Studies accepting inclusion beyond $48 \mathrm{~h}$ from the onset of symptoms were excluded. The last database search was conducted on September 2019. Following screening of abstracts, fulltext copies of potentially eligible papers were retrieved and assessed for eligibility.

\section{Results}

Electronic database search yielded 32 publications and 27 clinical trials of which 19 studies were finally included in the systematic literature review (Fig. 2). Among the 31 publications identified on Pubmed search, nine articles were not related to stroke (29\%), four articles applied chronic PostRIC (13\%), three articles were reviews of literature, three articles described design of the studies or protocols [14-16], two articles were on subarachnoid hemorrhage patients, one article was a sub-study and four articles were not eligible. After applying the inclusion criteria (acute ischemic stroke-AIS patients and application of remote ischemic perconditioning-RIPerC) and the studies that accept inclusion beyond $48 \mathrm{~h}$ from the onset of symptoms were exclude; a total of 6 articles were included and analyzed in the systematic review [16-21], note that 4 out of the 6 papers were previously registered as clinical trials [18-21]. Twenty-seven randomized clinical trials (RCTs) were identified on clinicaltrials.gov. Of these RCTs, 6 (22.2\%) applied PostRIC and 4 (14.8\%\%) were not considered after inclusion/exclusion criteria were applied. 


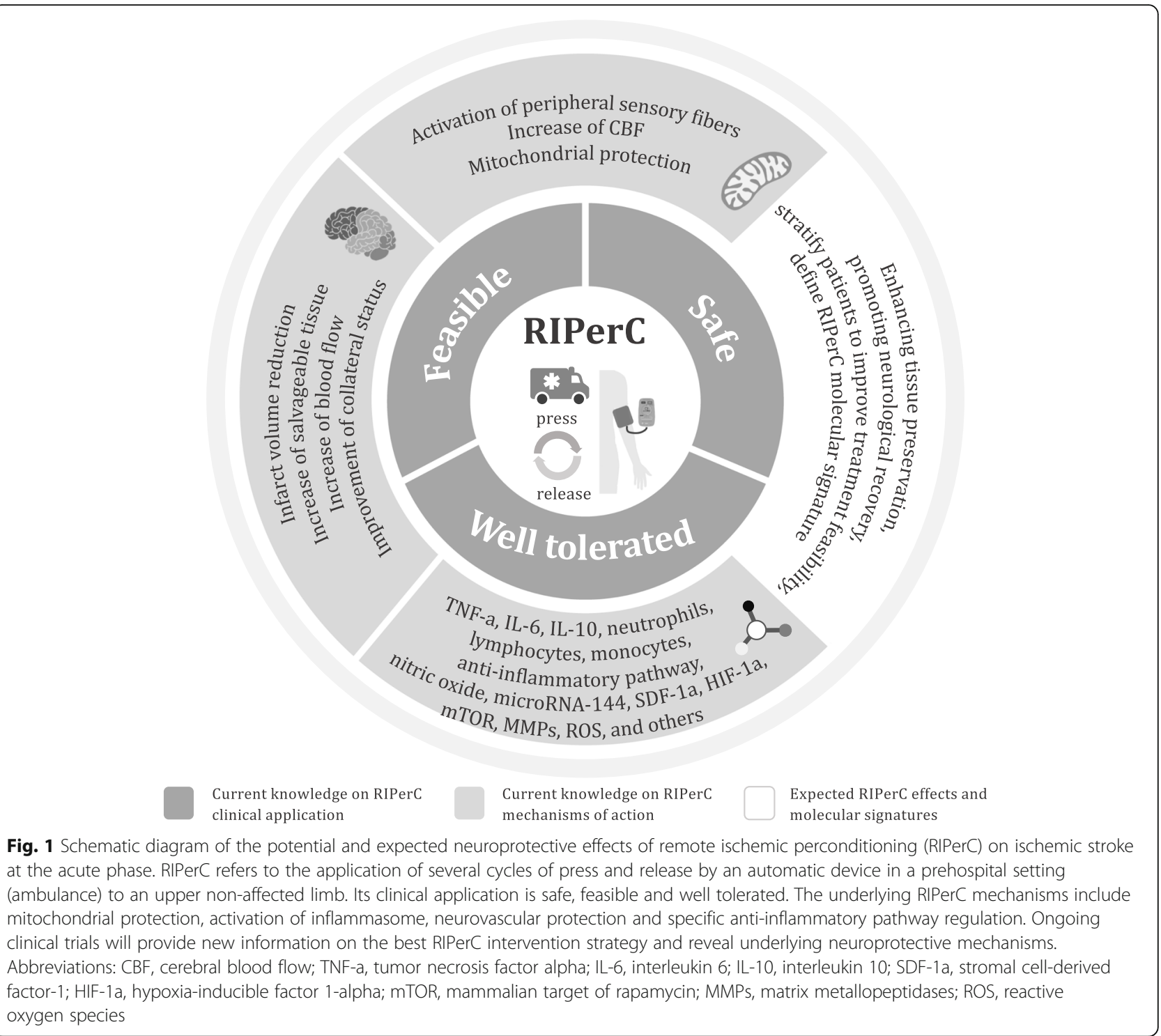

Seventeen RCTs were further considered in the present systematic review (NCT0097596 [21], RESCUE-BRAIN [15, 22], REVISE-1 [18], rtPA-RIC1 [19], ReCAST-2, rtPA-RIC, REMOTE-CAT, TRIPCAIS, REVISE-2, RICE PAC, SERIC-AIS, RICAMIS, RESIST [14], ICARUS, SERICT-AIS, RIC-SIID, PROTECT I). Table 1 provides a summary of study design characteristics of the 19 RTCs on RIPerC application on IS patients.

The first research paper was published by Hougard et al. in 2014 [21]. Of 443 randomized patients, 247 received manual remote ischemic conditioning (mRIC) during transportation in the ambulance to the hospital. After adjustment for baseline multimodal magnetic resonance imaging (MRI) findings, voxel-wise logistical analysis showed better radiological evolution of mRIC treated patients than non-treated patients. However, there were no significant differences in clinical neurological outcome between mRIC and control groups. The paper of Che et al. [19], included only 30 patients treated with recombinant tissue plasminogen activator (rt-PA). Zhao et al. [18] demonstrated that RIC is safe in 20 patients who underwent mechanical thrombectomy. Moreover, England et al. [17] confirmed the applicability and feasibility of RIC on 13 IS patients within $24 \mathrm{~h}$ after the onset of symptoms. Furthermore, RIC was associated with changes of plasma biomarkers related to ischemic tolerance (IT) phenomena, such as HSP27 and phosphorylated HSP27, whose expression was significantly different when both arms (control vs. experimental) of the trial were compared $(n=13)$ [17]. These four publications included a limited and small number of recruited subjects [17-19, 21]. In contrast with previous studies, the multicenter RESCUE-BRAIN trial [20] was not only focused on IS patients who 


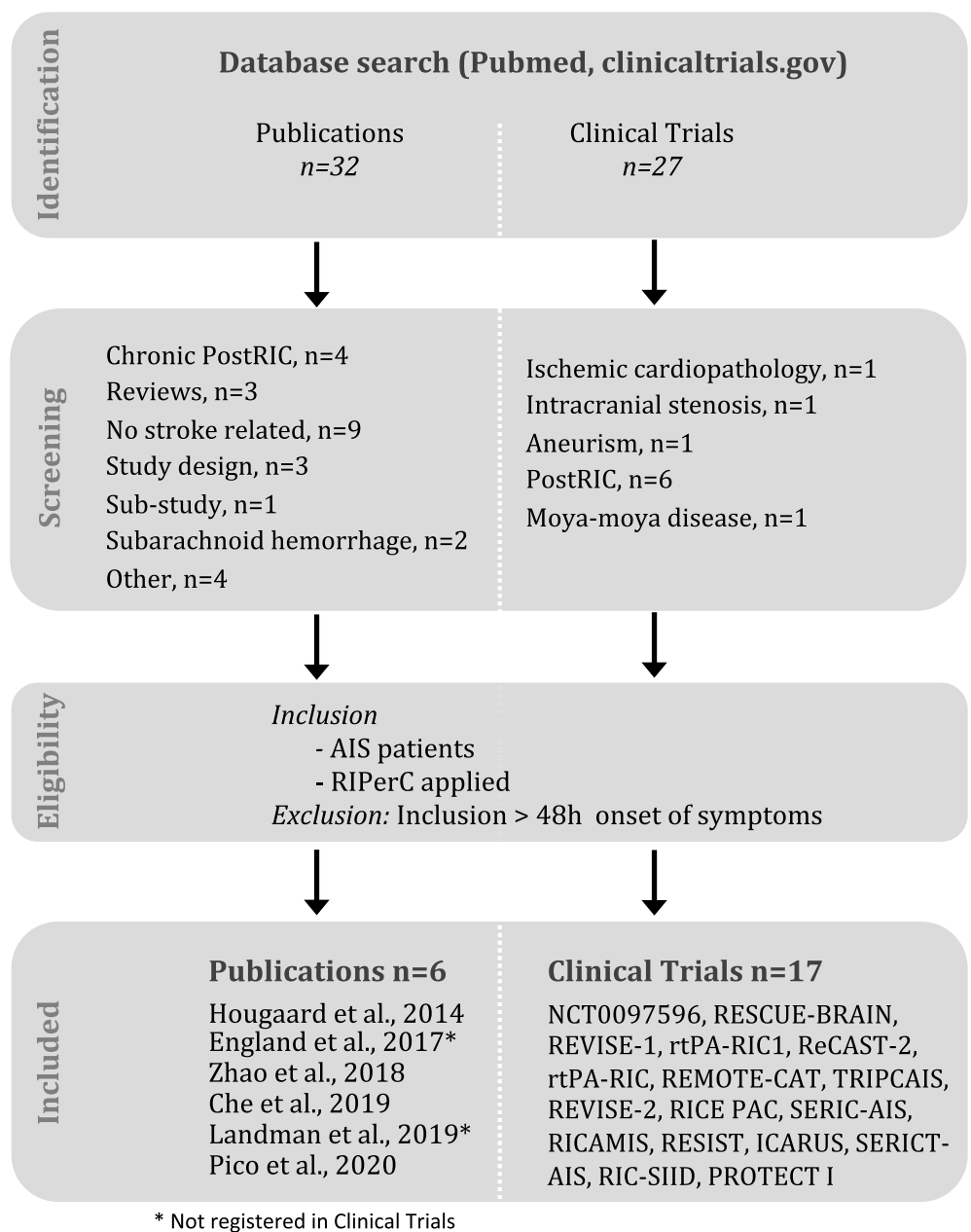

Fig. 2 PRISMA diagram details the search and selection process of RIPerC systematic review of literature

received or were candidate for revascularization therapies. It included 188 patients with confirmed carotid IS who underwent magnetic resonance imaging within $6 \mathrm{~h}$ after the onset of symptoms, and 171 (91\%) patients received a recanalization therapy. In RESCUE_BRAIN trial, RIPerC was applied using an electronic device on the unaffected lower extremity (4 cycles of 5-min inflations and 5-min deflations). Brain infarction volume growth, which was the main outcome, was not significantly different between the intervention and control groups. In addition, no significant differences at 90-days mRS and mortality were observed between the two groups.

Up to now, there are 19 RCTs identified (where?) and $17(89.5 \%)$ of them were registered in clinicaltrials.gov. Among them, 14 (73.4\%) have been registered in the last 3 years, 9 (47.4\%) have been developed in China, $9(47.4 \%)$ in Europe and one (5.3\%) in United States. Relating to the estimated number of enrolled patients on selected RTCs, special attention must be paid on RICAMIS $(n=1800)$, RESIST $(n=1500,14)$, SERIC-AIS $(n=912)$ and REMOTE-CAT $(n=572)$.

There is a high variability in the inclusion and exclusion criteria among trials. Five RCTs require radiological confirmation of acute cerebral infarction despite of the subsequent treatment received (SERIC-AIS, RIC-SHD, RICAMIS, RECAST, RESCUE BRAIN). Finally, Danish RESIST RCTs, Spanish REMOTE-CAT and British RECAST-2 include patients that met stroke code criteria. Both REMOTE-CAT and RESIST consider the score of prehospital scales: RACE scale [23] and Prehospital Stroke Score (PreSS), respectively. Only 6 trials (31.6\%) set up an upper age limit as an inclusion criterion. Like in previous RCTs of Hougard et al. [21] and Che et al. [19], three on-going RCTs (SERICT-AIS, rtPA-RIC, TRIPCAIS) are focused on the RIC's role as an adjuvant treatment of thrombolytic therapy. In contrast, REVISE-2, PROTECT I and REVISE-1 [18] included patients who underwent thrombectomy. 


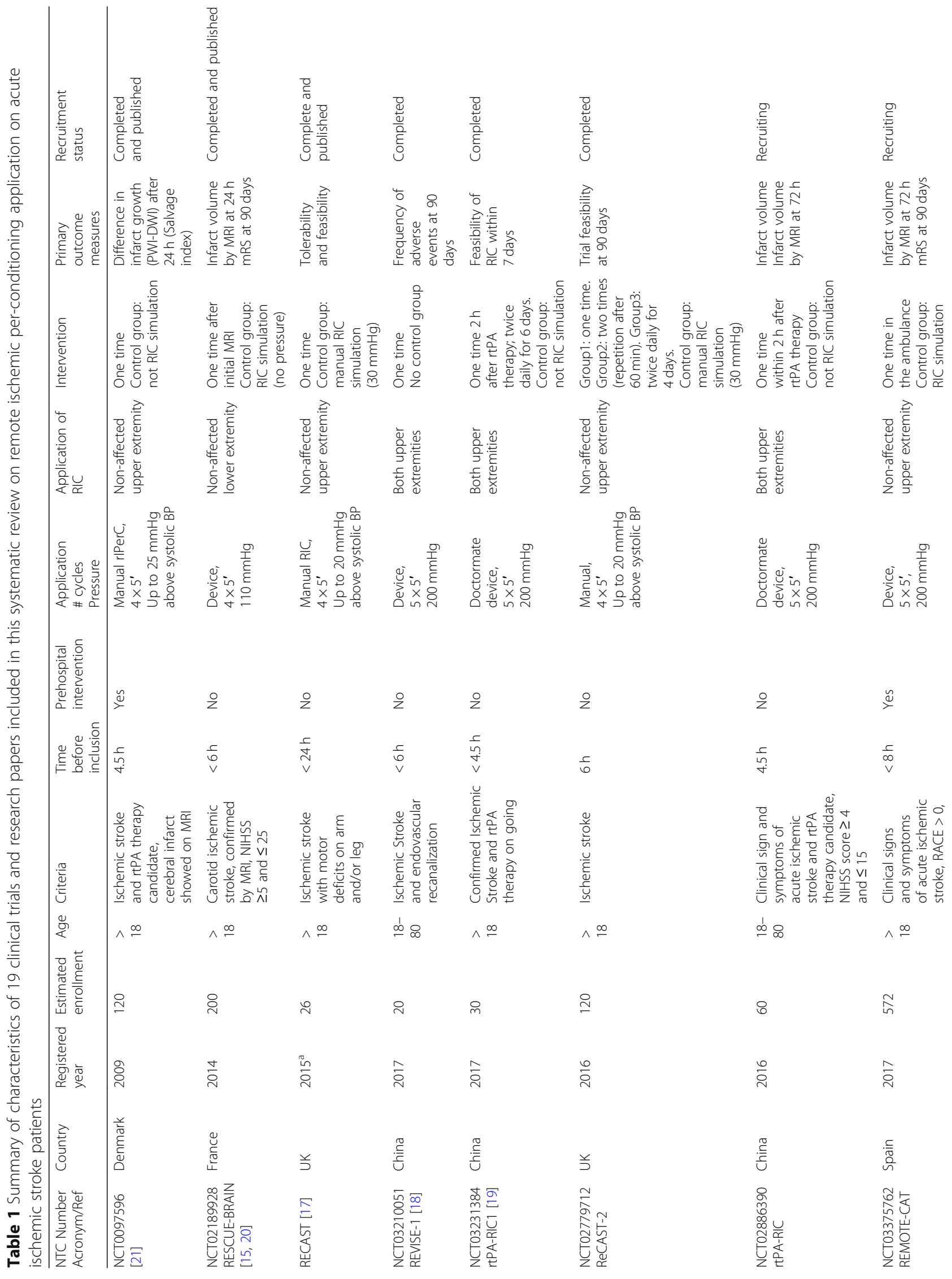


Purroy et al. BMC Neurology

(2020) 20:266

Page 6 of 12

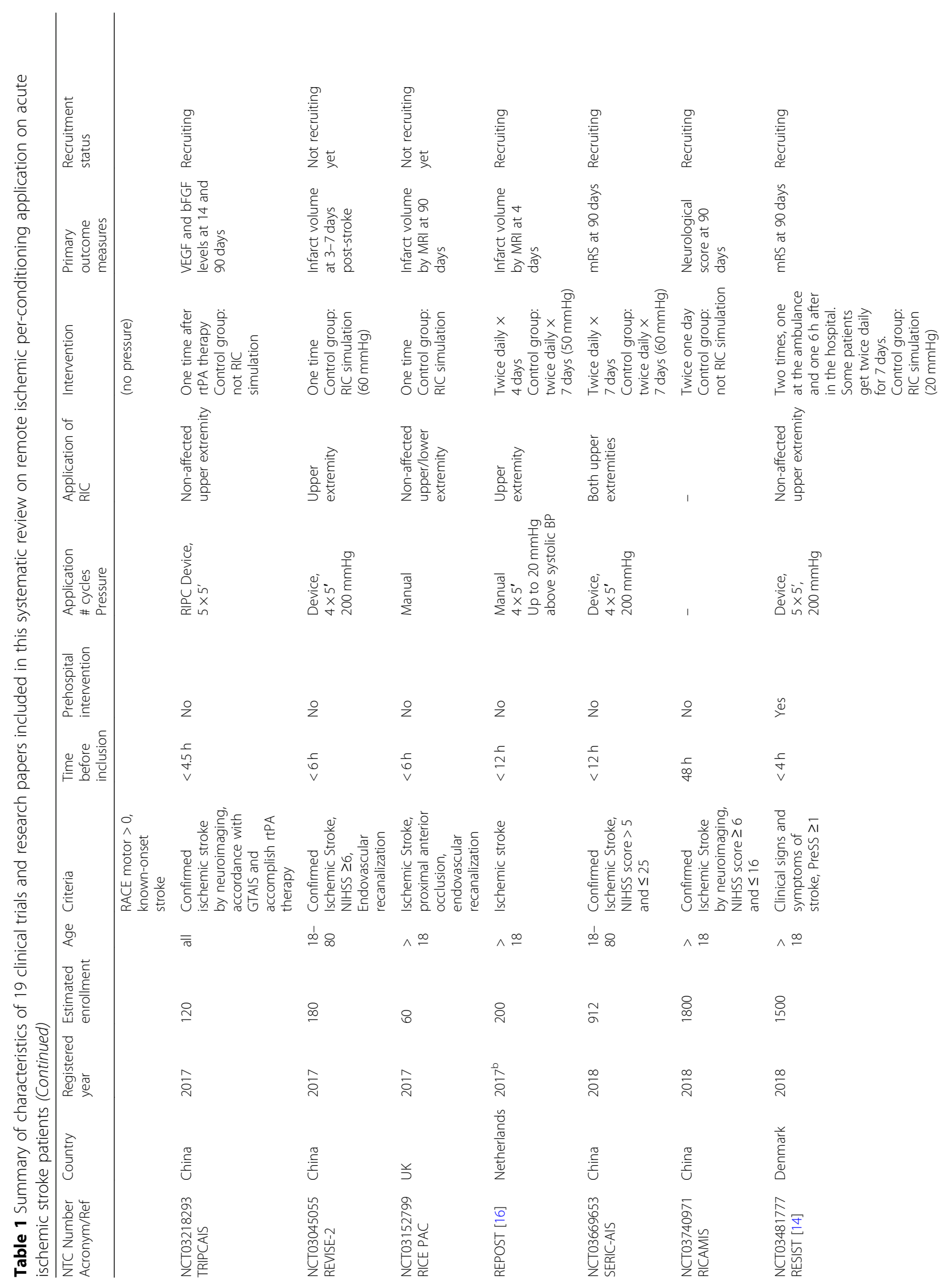


Purroy et al. BMC Neurology

(2020) 20:266

Page 7 of 12

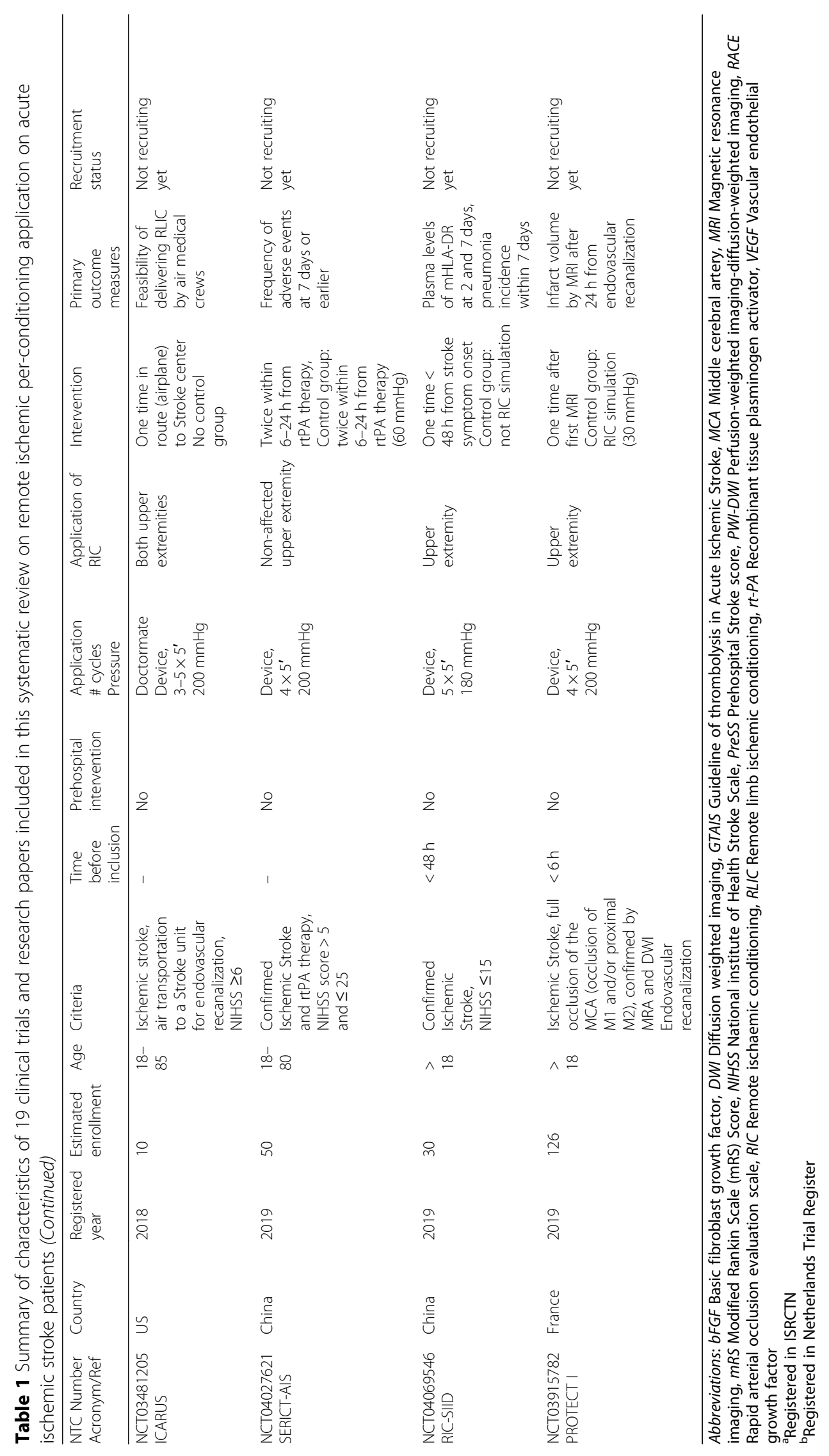


Heterogeneity is also evidenced by the number of RIC cycles applied: 7 (36.8\%) RCTs use 5 cycles, one $(5.3 \%)$ RCT uses between 3 and 5 cycles, and the rest of the trials use 4 cycles. Thirteen $(68.4 \%)$ RCTs perform a single application of RIC. Conversely, SERIC-AIS and RESIST [14] have planned up to two applications throughout 7 days, like in the finished study of Che et al. [19]; only REPOST has planned to applied during 4 days [16]. The application of RIC is located in the non-paretic lower limb only in one RCT [15], on both upper extremities in five (26.3\%) RCTs, and on upper or lower non-paretic extremities in one (5.3\%) RCT. In most cases, the application is restricted to the unaffected upper limb. The application of the RIC is manual in 5 (26.3\%) RCTs: two completed RCT [17, 21], REPOST [16], RECAST 2 and RICE PAC. A simulated control group is only included in little over half of the considered RCTs.

Certain variability of timing of RIC application is observed within all selected studies. Concretely, in the RESIST trial, temporal inclusion criterion is set at $<4 \mathrm{~h}$ while in RIC-SIID and RICAMIS is extended to $48 \mathrm{~h}$. RCTs focused on patients treated with intravenous fibrinolysis set the maximum time for the evolution of symptoms to $4.5 \mathrm{~h}$. Instead, among RCTs assessing the effect of RIC on thrombectomy, the time is set up at $6 \mathrm{~h}$. The Spanish REMOTE-CAT trial includes patients with less than $8 \mathrm{~h}$ of evolution of symptoms.

Only three RCTs, REMOTE-CAT, RESIST and the previous published by Hougard et al. [21], initiate the application of RIC in a prehospital setting, usually in the ambulance transportation of the patient to the hospital or stroke care center. Despite the low sample size $(n=$ 15), ICARUS trial aims to reveal the feasibility of RIC application on thrombectomy candidates who are transported to comprehensive stroke centers by aircraft.
$\mathrm{C}$, outcome measurements, was there any information on the size of the final infarct volume, perfusion, recurrent stroke?

The high heterogeneity within RCTs is also observed on the main endpoints (Fig. 3) and outcome measurements. The RCTs yielding the highest number of enrolled patients are still on-going (REMOTE-CAT, SERIC AIS, RESIT and RICAMIS) and all have considered the clinical endpoint as the main endpoint. In medium size studies and endovascular therapy related studies, the main endpoints are infarct volume and/or neuroimaging outputs. On the first research published paper on the application of RIC on IS patients, the main endpoint considered was the neuroimaging outcome [21]. Ischemic tolerance-related biomarkers are included in TRIPCAIS and RIC-SIID trials. However, other RCTs would also study biomarkers to detect differential expression changes. Small-size recruited patients studies demonstrate whether RIC application is feasible in AIS patients and AIS patients treated with rt-PA and/or endovascular therapy [17-19] (Fig. 3).

\section{Discussion}

The current systematic review of RIPerC in IS patients has revealed a noticeable number of trials registered in clinicaltrials.gov, especially in the last 3 years. Globally, a broad heterogeneity is observed among RCTs regarding the number of recruited patients, inclusion criteria, number of RIPerC applied cycles, location of the application, and the main endpoints. Despite the high heterogeneity of current studies, they would all contribute to improve RIPerC effects and mechanisms of action. The first published evidence of RIPerC in IS patients was limited to patients that underwent intravenous alteplase therapy [21]. Moreover, according to new advances in

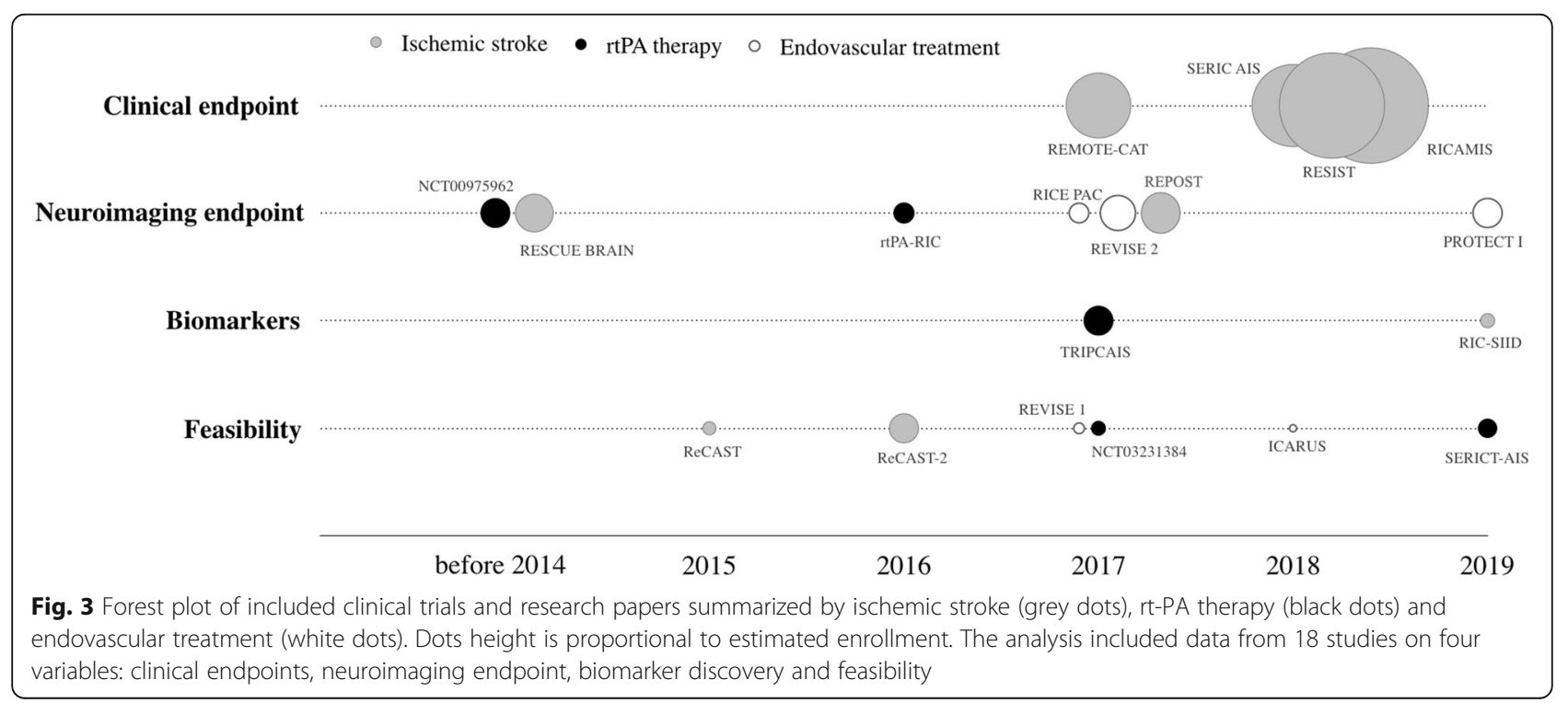


stroke, five new studies have been focused on patients treated with endovascular therapy. However, preclinical data have demonstrated that RIC during acute ischemia is effective when applied both alone and in combination with revascularization therapies [24]. For that, results of the largest RCTs (REMOTE-CAT, SERIC AIS, RESIST [14] and RICAMIS), which all include IS patients despite of the acute applied treatment, would be of enormous interest.

Only one RCT applied RIC manually [21], but one out of three patients fully complete the cycles. Using an automated RIC device allows that RIC can be continued once the patient arrives to the stroke care unit and the full dose can be administered. For that, most of the RTCs are currently using automatic devices to apply RIPerC. Concretely, 14 out of 17 new trials use automatic devices. Another important issue is the number of cycles and the place of application. Most RIC trials in Cardiology $[25,26]$ and the first trials in IS used the four-cycle protocol, probably due to literature tradition and preclinical studies. Preconditioning was first demonstrated in a dog model of myocardial ischemia using a four-cycle protocol [27]. Afterwards, both RIC before ischemia [28] and RIC during ischemia were first documented using the same protocol [29]. The neutral clinical results of Hougaard et al. [21] and Pico et al. [20] trials arise the need to increase the RIC stimulus and repetitions. Recent studies in preclinical models also addressed it to optimize the efficacy and optimal duration of RIPerC [30]. In a rat model of cerebral ischemia, repeated remote post-conditioning during 14 days after reperfusion significantly decrease the volume of infarction [31]. There are some promising experiences in chronic postconditioning among intracranial stenosis patients [32] and patients with cerebral small-vessel disease [33] using five-cycle protocol. Currently, on-going REMOTE-CAT and RESIST trials use a five-cycle RIC protocol. Moreover, combination of RIPerC and postconditioning during 4 to 7 days is assessed in the RESIST trial [14], ReCAST-2 [17], REPOST [16] and SERIC-AIS trial. Although, the volume of muscle mass affects the efficacy of the RIC intervention [34], only one study proposed RIC application in a leg [20]. It has been described that one in four IS patients has silent peripheral arterial disease [35], for that it has suggested that the upper arm would be the best location because of safety reasons. One and two-limb conditioning were equally protective according to preclinical models [30]. At present, how the neuroprotective stimulus is transferred or its mechanisms of actions in the brain are not fully understood [36], but it is known that the translation of the RIC sensory signal to the brain is crucial [37] and RIC should be applied in the non-affected arm.
A prehospital administration of RIC in the ambulance transportation was first proposed by Hougard et al. [21] and it is established in REMOTE-CAT and RESIST [14] trials. RIC effects are time-dependent, so early initiation of RIC is fundamental [38].

Pre-hospital screening scales should be used during transportation and RIPerC application to correctly randomize and recruit IS patients. When the Face Arm Speech Test was used, an increased proportion of patients with transient symptoms in the intervention group was observed [21]. It was not clear whether it was a RIC's effect or there was a bias in the selection. At present, both REMOTE-CAT and RESIST [14] trials have a pre-hospital screening performed by RACE and PreSS scores, respectively. Patients should be properly balanced using prehospital stroke scores.

Recently, it has been reported that RIC improves the clinical evolution of myocardial infarction and it reduces the final lesion size [25, 39]; but a recent large RCT, with more than 5000 patients, reported no effects on clinical outcomes [40]. Cerebral and heart ischemia might differ on its own characteristics [41], because IS has a variety of pathogenic mechanisms not present in heart ischemia. The rupture or erosion of vulnerable plaques in coronary arteries are the most common cause of heart ischemia [42], while the embolism from arterial or heart sources is the main cause of IS [43]. Altogether, we would anticipate that underlying RIC mechanisms and clinical outcomes in IS patients will be different and the expected results of the currents RCTs are promising.

\section{Implications for future research}

Currently, there are some on-going randomized clinical trials that will provide valuable information on RIPerC in ischemic stroke patients. However, future studies should carefully examine patient recruitment, RIPerC application settings, proper outcome measurements and neuroimaging follow-up protocols. All optimization and efforts will improve the current knowledge and address new medical strategies and management of stroke patients.

According to the RESCUE BRAIN study [20], the application of RIC during/after partial or complete reperfusion was futile, and it did not reduce the consequences of reperfusion injury. So, this might suggest that RIC should be applied differently. In this line, preclinical data and results from pilot studies showed that RIC should be applied as soon as possible, preferable during patient transportation (prehospital setting, ambulance) to a Hospital, in order to avoid the penumbral tissue recruitment and extend the time window for further application of reperfusion therapies. In this context, an early triage and stratification of the patients using prehospital scales are essential, and it will also help in the randomization 
process of the clinical trials (REMOTE-CAT, NCT03375762; RESIST [14]). The accuracy of prehospital scales is fundamental to identify or confirm a possible early prehospital treatment effect, like it was suspected in previous studies [21]. For that, the initial use of prehospital scales is a strong recommendation along RIPerC application in a prehospital setting and/or as soon as stroke symptoms are detected.

Automatic devices should be used to ensure completion of cycles and to document the treatment compliance. Another reason for the futile results of RESCUE BRAIN [20] study and the study of Hougaard et al. [21] would be that the $4 \times 5$ cycles of RIPerC stimulus was not sufficient. To overcome this issue, increasing up to five cycles and/or the stimulus repetition twice daily for the first 5 to 7 days would be an improvement. In the other hand, better selection of included patients in clinical trials can boost patient stratification.

Collateral status correlates with stroke severity and reperfusion outcomes, due to their ability to restrict the growth of penumbral territory [44]. Although the underlying mechanisms of RIC are still not fully known, some recent preclinical studies have showed an enhancement of collateral circulation [45, 46]. For that, the role of collaterals is essential in large vessel occlusion (LVO) patients, whom are also candidates to undergo mechanical thrombectomy $[47,48]$ in admitted hospital, or they are candidates to be transferred to a Comprehensive Stroke center. Altogether, LVO patients would be a group of special interest to study the RIC effects.

Recent published data have highlighted that RIC is safe and feasible [17-21, 49] similarly to RCTs involving patients with myocardial infarction [25]. For that, the main outcomes of the ongoing and future RCTs on RIPerC have a strong clinical interest. According to stroke treatment academic industry roundtable (STAIR) recommendations [50], 24-h NIHSS, 7-days mRS and 90-days mRS should be considered to be the standard clinical endpoints in acute stroke trials. Follow-up infarct volume on brain imaging is also informative, based on preclinical data that reported an effect of RIC on final brain infarction volume when it was used alone or in combination with alteplase [24]. This is recommended by both STAIR [50] and The Stroke Imaging Research (STIR) group [47]. More concretely, STIR estimated that sample sizes based on lesion volumes should be about one fourth of those based on mRS [51], so the imaging endpoint has the advantage of requiring smaller sample size. Finally, the understanding of RIPerC mechanisms and its neuroprotective role will be a key and animal models studies surely encourage better refined and translation into humans for the treatment of ischemic stroke.

\section{Conclusions}

The summary of the completed and ongoing RCTs on RIPerC in IS patients shows that RIC can be initiated during pre-hospital transport, and it can be used alone or in combination with current recanalization therapies. RIPerC has the advantages of simplicity, safety, feasibility and affordability. The exact time window and the most effective neuroprotective RIC protocol are still not fully determined. Stroke preclinical animal models and RIC research are needed, both will also contribute to define the RIC molecular effects. Finally, ongoing RCTs will provide new information on the effect of RIPerC in IS patients, the optimal RIC protocol application and the underlying RIPerC mechanisms.

\section{Abbreviations \\ IS: Ischemic stroke; IT: Ischemic tolerance; mRIC: Manual remote ischemic conditioning; RCT: Randomized clinical trials; RIC: Remote ischemic conditioning; RIPerC: Remote ischemic preconditioning; Rt-PA: Recombinant tissue plasminogen activator; VEGF: Vascular endothelial growth factor}

\section{Acknowledgements}

Not applicable.

\section{Authors' contributions}

FP, CG, GM, CP, CT, DV-J, MV-P, AV and GA have made substantial contributions to the design of the review. FP and GA have analyzed the data. FP and GA have written the paper. CG, GM, CP, CT, DV-J, MV-P and AV substantively revised the manuscript. All authors read and approved the final manuscript.

\section{Funding}

This study was supported by the Government of Catalonia-Agència de Gestió d'Ajuts Universitaris i de Recerca (FP: 2017 SGR 1628), Instituto de Salud Carlos III and co-funded by European Union (ERDF/ESF, "A way to make Europe") (FP: Project PI17-01725) and the INVICTUS plus Research Network (Carlos III Health Institute).

These funding sources had no role in the design of the study and collection, analysis, and interpretation of data and in writing the manuscript.

\section{Availability of data and materials}

The data that support the findings of this study are available from the corresponding author upon reasonable request.

Ethics approval and consent to participate

Not applicable.

\section{Consent for publication}

Not applicable.

\section{Competing interests}

The authors declare that they have no competing interests.

Received: 16 January 2020 Accepted: 22 June 2020

Published online: 02 July 2020

\section{References}

1. Mozaffarian D, Benjamin EJ, Go AS, Arnett DK, Blaha MJ, Cushman M, et al. Executive summary: heart disease and stroke statistics--2016 update: a report from the American Heart Association. Circulation. 2016;133(4):447-54.

2. Pandian JD, Gall SL, Kate MP, Silva GS, Akinyemi RO, Ovbiagele BI, et al. Prevention of stroke: a global perspective. Lancet. 2018;392(10154):1269-78.

3. Purroy F, Vena A, Forne C, de Arce AM, Davalos A, Fuentes B, et al. Age- and sex-specific risk profiles and in-hospital mortality in 13,932 Spanish stroke patients. Cerebrovasc Dis. 2019:47(3-4):151-64

4. Lees KR, Emberson J, Blackwell L, Bluhmki E, Davis SM, Donnan GA, et al. Effects of Alteplase for acute stroke on the distribution of functional outcomes: a pooled analysis of 9 trials. Stroke. 2016;47(9):2373-9. 
5. Emberson J, Lees KR, Lyden P, Blackwell L, Albers G, Bluhmki E, et al. Effect of treatment delay, age, and stroke severity on the effects of intravenous thrombolysis with alteplase for acute ischaemic stroke: a meta-analysis of individual patient data from randomised trials. Lancet. 2014;384(9958):1929_ 35.

6. Goyal M, Menon BK, van Zwam WH, Dippel DW, Mitchell PJ, Demchuk AM, et al. Endovascular thrombectomy after large-vessel ischaemic stroke: a meta-analysis of individual patient data from five randomised trials. Lancet. 2016;387(10029):1723-31.

7. Chamorro A, Dirnagl U, Urra X, Planas AM. Neuroprotection in acute stroke: targeting excitotoxicity, oxidative and nitrosative stress, and inflammation. Lancet Neurol. 2016;15(8):869-81.

8. O'Collins VE, Macleod MR, Donnan GA, Horky LL, van der Worp BH, Howells DW. 1,026 experimental treatments in acute stroke. Ann Neurol. 2006;59(3): 467-77.

9. Percie du Sert N, Alfieri A, Allan SM, Carswell HV, Deuchar GA, Farr TD, et al. The IMPROVE guidelines (Ischaemia models: procedural refinements of in vivo experiments). J Cereb Blood Flow Metab. 2017;37(11):3488-517.

10. Hausenloy DJ, Yellon DM. The therapeutic potential of ischemic conditioning: an update. Nat Rev Cardiol. 2011;8(11):619-29.

11. Hess DC, Blauenfeldt RA, Andersen G, Hougaard KD, Hoda MN, Ding Y, et al. Remote ischaemic conditioning-a new paradigm of self-protection in the brain. Nat Rev Neurol. 2015;11(12):698-710.

12. Gidday JM. Cerebral preconditioning and ischaemic tolerance. Nat Rev Neurosci. 2006;7(6):437-48

13. Moher D, Liberati A, Tetzlaff J, Altman DG, Group P. Preferred reporting items for systematic reviews and meta-analyses: the PRISMA statement. J Clin Epidemiol. 2009:62(10):1006-12.

14. Blauenfeldt RA, Hjort N, Gude MF, Behrndtz AB, Fisher M, Valentin JB, et al. A multicentre, randomised, sham-controlled trial on REmote iSchemic conditioning in patients with acute STroke (RESIST) - rationale and study design. Eur Stroke J. 2020;5(1):94-101.

15. Pico F, Rosso C, Meseguer E, Chadenat ML, Cattenoy A, Aegerter P, et al. A multicenter, randomized trial on neuroprotection with remote ischemic perconditioning during acute ischemic stroke: the REmote iSchemic Conditioning in acUtE BRAin INfarction study protocol. Int J Stroke. 2016; 11(8):938-43.

16. Landman $T$, Schoon $Y$, Warle M, De Leeuw FE, Thijssen D. The effect of repeated remote ischemic postconditioning on infarct size in patients with an ischemic stroke (REPOST): study protocol for a randomized clinical trial. Trials. 2019;20(1):167.

17. England TJ, Hedstrom A, O'Sullivan S, Donnelly R, Barrett DA, Sarmad S, et al. RECAST (remote ischemic conditioning after stroke trial): a pilot randomized placebo controlled phase II trial in acute ischemic stroke. Stroke. 2017:48(5):1412-5.

18. Zhao W, Che R, Li S, Ren C, Li C, Wu C, et al. Remote ischemic conditioning for acute stroke patients treated with thrombectomy. Ann Clin Transl Neurol. 2018;5(7):850-6.

19. Che $R$, Zhao $W, M a ~ Q$, Jiang $F, W u L, Y u Z$, et al. rt-PA with remote ischemic postconditioning for acute ischemic stroke. Ann Clin Transl Neurol. 2019; 6(2):364-72.

20. Pico F, Lapergue B, Ferrigno M, Rosso C, Meseguer E, Chadenat ML, et al. Effect of in-hospital remote ischemic perconditioning on brain infarction growth and clinical outcomes in patients with acute ischemic stroke: the RESCUE BRAIN randomized clinical trial. JAMA Neurol. 2020;77(6):1-11.

21. Hougaard KD, Hjort N, Zeidler D, Sørensen L, Nørgaard A, Hansen TM, et al. Remote ischemic perconditioning as an adjunct therapy to thrombolysis in patients with acute ischemic stroke: a randomized trial. Stroke. 2014;45(1): 159-67.

22. Pico F, Laperque M, Ferrigno M, Chadenat ML, Bourdain F, Meseguer E, et al. The RESCUE BRAIN trial: a french multicenter randomized trial on neuroprotection with lower limb ischemic per-conditioning in the acute phase of cerebral infarction. Eur Stroke J. 2019;4(1 S):3.

23. Perez de la Ossa N, Carrera D, Gorchs M, Querol M, Millan M, Gomis M, et al. Design and validation of a prehospital stroke scale to predict large arterial occlusion: the rapid arterial occlusion evaluation scale. Stroke. 2014:45(1):8791.

24. Hoda MN, Siddiqui S, Herberg S, Periyasamy-Thandavan S, Bhatia K, Hafez SS, et al. Remote ischemic perconditioning is effective alone and in combination with intravenous tissue-type plasminogen activator in murine model of embolic stroke. Stroke. 2012;43(10):2794-9.
25. Man C, Gong D, Zhou Y, Fan Y. Meta-analysis of remote ischemic conditioning in patients with acute myocardial infarction. Sci Rep. 2017;7: 43529.

26. Pryds K, Nielsen RR, Jorsal A, Hansen MS, Ringgaard S, Refsgaard J, et al. Effect of long-term remote ischemic conditioning in patients with chronic ischemic heart failure. Basic Res Cardiol. 2017;112(6):67.

27. Murry CE, Jennings RB, Reimer KA. Preconditioning with ischemia: a delay of lethal cell injury in ischemic myocardium. Circulation. 1986;74(5):1124-36.

28. Birnbaum Y, Hale SL, Kloner RA. Ischemic preconditioning at a distance: reduction of myocardial infarct size by partial reduction of blood supply combined with rapid stimulation of the gastrocnemius muscle in the rabbit. Circulation. 1997:96(5):1641-6.

29. Schmidt MR, Smerup M, Konstantinov IE, Shimizu M, Li J, Cheung M, et al. Intermittent peripheral tissue ischemia during coronary ischemia reduces myocardial infarction through a KATP-dependent mechanism: first demonstration of remote ischemic perconditioning. Am J Physiol Heart Circ Physiol. 2007;292(4):H1883-90.

30. Johnsen J, Pryds K, Salman R, Lofgren B, Kristiansen SB, Botker HE. The remote ischemic preconditioning algorithm: effect of number of cycles, cycle duration and effector organ mass on efficacy of protection. Basic Res Cardiol. 2016:111(2):10.

31. Ren C, Wang P, Wang B, Li N, Li W, Zhang C, et al. Limb remote ischemic per-conditioning in combination with post-conditioning reduces brain damage and promotes neuroglobin expression in the rat brain after ischemic stroke. Restor Neurol Neurosci. 2015;33(3):369-79.

32. Meng R, Asmaro K, Meng L, Liu Y, Ma C, Xi C, et al. Upper limb ischemic preconditioning prevents recurrent stroke in intracranial arterial stenosis. Neurology. 2012;79(18):1853-61.

33. Wang Y, Meng R, Song H, Liu G, Hua Y, Cui D, et al. Remote ischemic conditioning may improve outcomes of patients with cerebral small-vessel disease. Stroke. 2017;48(11):3064-72.

34. Hess DC, Hoda MN, Bhatia K. Remote limb perconditioning [corrected] and postconditioning: will it translate into a promising treatment for acute stroke? Stroke. 2013:44(4):1191-7.

35. Purroy F, Coll B, Oro M, Seto E, Pinol-Ripoll G, Plana A, et al. Predictive value of ankle brachial index in patients with acute ischaemic stroke. Eur J Neurol. 2010;17(4):602-6.

36. Heusch G, Botker HE, Przyklenk K, Redington A, Yellon D. Remote ischemic conditioning. J Am Coll Cardiol. 2015;65(2):177-95.

37. Donato F, Rompani SB, Caroni P. Parvalbumin-expressing basket-cell network plasticity induced by experience regulates adult learning. Nature. 2013;504(7479):272-6

38. Koch S, Gonzalez N. Preconditioning the human brain: proving the principle in subarachnoid hemorrhage. Stroke. 2013;44(6):1748-53.

39. Botker HE, Kharbanda R, Schmidt MR, Bottcher M, Kaltoft AK, Terkelsen CJ, et al. Remote ischaemic conditioning before hospital admission, as a complement to angioplasty, and effect on myocardial salvage in patients with acute myocardial infarction: a randomised trial. Lancet. 2010;375(9716): 727-34

40. Hausenloy DJ, Kharbanda RK, Moller UK, Ramlall M, Aaroe J, Butler R, et al. Effect of remote ischaemic conditioning on clinical outcomes in patients with acute myocardial infarction (CONDI-2/ERIC-PPCI): a single-blind randomised controlled trial. Lancet. 2019:394(10207):1415-24.

41. Soler EP, Ruiz VC. Epidemiology and risk factors of cerebral ischemia and ischemic heart diseases: similarities and differences. Curr Cardiol Rev. 2010; 6(3):138-49

42. White HD, Chew DP. Acute myocardial infarction. Lancet. 2008;372(9638): 570-84.

43. Campbell BCV, De Silva DA, Macleod MR, Coutts SB, Schwamm LH, Davis SM, et al. Ischaemic stroke. Nat Rev Dis Primers. 2019;5(1):70.

44. Kimmel ER, Al Kasab S, Harvey JB, Bathla G, Ortega-Gutierrez S, Toth G, et al. Absence of collaterals is associated with larger infarct volume and worse outcome in patients with large vessel occlusion and mild symptoms. J Stroke Cerebrovasc Dis. 2019:28(7):1987-92.

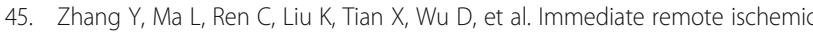
postconditioning reduces cerebral damage in ischemic stroke mice by enhancing leptomeningeal collateral circulation. J Cell Physiol. 2019;234(8): 12637-45.

46. Kitagawa K, Saitoh M, Ishizuka K, Shimizu S. Remote limb ischemic conditioning during cerebral ischemia reduces infarct size through 
enhanced collateral circulation in murine focal cerebral ischemia. J Stroke Cerebrovasc Dis. 2018;27(4):831-8.

47. Warach SJ, Luby M, Albers GW, Bammer R, Bivard A, Campbell BC, et al. Acute stroke imaging research roadmap III imaging selection and outcomes in acute stroke reperfusion clinical trials: consensus recommendations and further research priorities. Stroke. 2016:47(5):1389-98.

48. Berkhemer OA, Jansen IG, Beumer D, Fransen PS, van den Berg LA, Yoo AJ, et al. Collateral status on baseline computed tomographic angiography and intra-arterial treatment effect in patients with proximal anterior circulation stroke. Stroke. 2016;47(3):768-76.

49. Kate M, Brar S, George U, Rathore S, Butcher K, Pandian J, et al. Self- or caregiver-delivered manual remote ischemic conditioning therapy in acute ischemic stroke is feasible: the Early Remote Ischemic Conditioning in Stroke (ERICS) trial. Wellcome Open Res. 2019;4:147.

50. Jovin TG, Albers GW, Liebeskind DS, Consortium SI. Stroke treatment academic industry roundtable: the next generation of endovascular trials. Stroke. 2016;47(10):2656-65.

51. Whitehead J, Bolland K, Valdes-Marquez E, Lihic A, Ali M, Lees K, et al. Using historical lesion volume data in the design of a new phase II clinical trial in acute stroke. Stroke. 2009:40(4):1347-52.

\section{Publisher's Note}

Springer Nature remains neutral with regard to jurisdictional claims in published maps and institutional affiliations.

Ready to submit your research? Choose BMC and benefit from:

- fast, convenient online submission

- thorough peer review by experienced researchers in your field

- rapid publication on acceptance

- support for research data, including large and complex data types

- gold Open Access which fosters wider collaboration and increased citations

- maximum visibility for your research: over $100 \mathrm{M}$ website views per year

At BMC, research is always in progress.

Learn more biomedcentral.com/submissions 